\title{
The microbiology of pleural infection in adults: a systematic review
}

\author{
Maged Hassan (101,2,3 Tamsin Cargill (1) ${ }^{1}$, Elinor Harriss ${ }^{4}$, Rachelle Asciak ${ }^{1,2}$, \\ Rachel M. Mercer ${ }^{1,2}$, Eihab O. Bedawi (1) ${ }^{1,2}$, David J. McCracken ${ }^{1,2}$ \\ loannis Psallidas ${ }^{1,2}$, John P. Corcoran $\mathbb{1}^{5}$ and Najib M. Rahman ${ }^{1,2,6}$
}

Affiliations: ${ }^{1}$ Oxford Pleural Unit, Oxford University Hospitals, Oxford, UK. ${ }^{2}$ Oxford Respiratory Trial Unit, University of Oxford, Oxford, UK. ${ }^{3}$ Chest Diseases Dept, Alexandria Faculty of Medicine, Alexandria, Egypt. ${ }^{4}$ Bodleian Healthcare Libraries, University of Oxford, Oxford, UK. ${ }^{5}$ Interventional Pulmonology Service, Dept of Respiratory Medicine, University Hospitals Plymouth NHS Trust, Plymouth, UK. ${ }^{6}$ Oxford NIHR Biomedical Research Centre, Oxford, UK.

Correspondence: Maged Hassan, Oxford Pleural Unit and Oxford Respiratory Trials Unit, Churchill Hospital, Roosevelt Drive, OX3 7LE, Oxford, UK. E-mail: magedhmfagmail.com

@ERSpublications

Staphylococcus aureus is the predominant cause of pleural infection in adults with a temporal rise in rate of isolation of the drug-resistant organism. There are variations in the microbiology according to geographical location and setting of infection. http://bit.ly/31WnpI0

Cite this article as: Hassan M, Cargill T, Harriss E, et al. The microbiology of pleural infection in adults: a systematic review. Eur Respir J 2019; 54: 1900542 [https://doi.org/10.1183/13993003.00542-2019].

\begin{abstract}
Background and objectives: Pleural infection is a major cause of morbidity and mortality among adults. Identification of the offending organism is key to appropriate antimicrobial therapy. It is not known whether the microbiological pattern of pleural infection is variable temporally or geographically. This systematic review aimed to investigate available literature to understand the worldwide pattern of microbiology and the factors that might affect such pattern.

Data sources and eligibility criteria: Ovid MEDLINE and Embase were searched between 2000 and 2018 for publications that reported on the microbiology of pleural infection in adults. Both observational and interventional studies were included. Studies were excluded if the main focus of the report was paediatric population, tuberculous empyema or post-operative empyema.

Study appraisal and synthesis methods: Studies of $\geqslant 20$ patients with clear reporting of microbial isolates were included. The numbers of isolates of each specific organism/group were collated from the included studies. Besides the overall presentation of data, subgroup analyses by geographical distribution, infection setting (community versus hospital) and time of the report were performed.

Results: From 20980 reports returned by the initial search, 75 articles reporting on 10241 patients were included in the data synthesis. The most common organism reported worldwide was Staphylococcus aureus. Geographically, pneumococci and viridans streptococci were the most commonly reported isolates from tropical and temperate regions, respectively. The microbiological pattern was considerably different between communityand hospital-acquired infections, where more Gram-negative and drug-resistant isolates were reported in the hospital-acquired infections. The main limitations of this systematic review were the heterogeneity in the method of reporting of certain bacteria and the predominance of reports from Europe and South East Asia. Conclusions: In pleural infection, the geographical location and the setting of infection have considerable bearing on the expected causative organisms. This should be reflected in the choice of empirical antimicrobial treatment.
\end{abstract}

This article has supplementary material available from erj.ersjournals.com

This systematic review is registered at www.crd.york.ac.uk/prospero with registration number CRD42017076418.

Received: 16 March 2019 | Accepted after revision: 14 June 2019

Copyright @ERS 2019 


\section{Introduction}

Pleural infection is a common disease worldwide with considerable morbidity and mortality [1]. Despite improvements in healthcare quality, the incidence of pleural infection has been on the rise in the past two decades [1]. With the evidence supporting the use of fibrinolytics plus deoxyribonuclease (DNase) in management $[2,3]$ and widespread availability of less invasive (video-assisted thoracoscopic) surgery, the average cost per hospitalisation is currently estimated to be USD 4400 [4].

The cornerstones of treating pleural infection are prompt drainage of the infected fluid and timely initiation of antimicrobial treatment [5]. Antimicrobials are almost always started empirically with broad-spectrum coverage until microbial culture results inform more directed therapy. Commonly, the use of more focused and less broad spectrum antibiotics is not achievable, due to the yield of conventional cultures, which is in the vicinity of $40-60 \%$ [6]. Knowledge of the predominant organisms that cause pleural infection is a key and necessary step to achieve successful empirical coverage.

The so called "atypical" pathogens that commonly cause pneumonia do not have any significant role in pleural infection $[7,8]$, probably due to differences in the milieu between the lung parenchyma and the pleura. Recent reviews report the "milleri" group (more recently termed "Streptococcus anginosus group") of the Streptococcus genus as the most common culprit of pleural infection [6], but this is mainly based on data from Europe, North America and Australia. However, reports from Taiwan and South Korea cite Klebsiella species as the most common organism isolated in community-acquired pleural infections $[9,10]$.

This systematic review aimed to search the existing literature on the microbiology of pleural infection and determine the worldwide overall pattern of such microbiology. The primary research question was to assess the most common organisms/groups responsible for pleural infection in adults worldwide. The secondary research questions were to address 1) if there were different profiles of organisms according to geographical region; 2) the differences between the microbiology of hospital-acquired and community-acquired pleural infections; and 3) the average yield of microbial cultures in pleural infection.

\section{Methods}

\section{Protocol and registration}

The systematic review was performed in line with the Preferred Reporting Items for Systematic Reviews and Meta-Analyses (PRISMA) guidelines and its protocol was registered in the PROSPERO database (CRD42017076418).

\section{Search strategy and selection criteria}

This is a systematic review of papers published between 2000 and 2018 that report the microbiology of bacterial pleural infection in adults. No language restrictions were applied and non-English publications were included as long as reasonable translation could be obtained. All publications including data on microbiological results on pleural infection in adults were included. Studies with mixed adult and paediatric populations, but with predominance of adult patients $(>75 \%)$ were also included. Both observational (prospective and retrospective) and interventional studies were included provided that microbiological results were reported clearly. Reviews, conference abstracts and small case series reporting $<20$ subjects were excluded.

This systematic review focused on bacterial pleural infections. Reports describing pleural infection complicating thoracic surgery and spontaneous bacterial pleuritis were excluded as they were not felt to represent the typical microbiological pattern seen in the majority of cases with pleural infection. Articles with the main focus on tuberculous or fungal pleural infections were excluded, as these represent a different process to that seen in bacterial pleural infection. In addition, papers reporting exclusively on paediatric pleural infection or a single causative organism/group were excluded.

The initial electronic search was carried out on both Ovid Embase and MEDLINE databases for publications between 2000 and 2017 and was performed on July 31, 2017. The search was repeated on July 26, 2018 to include any newly published papers. The search terms used were "empyema", "pleural infection" and "pleuritis". The terms were intentionally broad to capture all publications. The full search strategy is available in the supplementary material.

\section{Screening and data management}

Due to the very large number of entries that the initial search returned, primary screening for relevant titles/abstracts was split between two authors (MH and TC) without duplication. The exclusion criteria of this phase are described in the Methods section. The second phase of screening to choose papers containing useful microbiological details was carried out independently by two reviewers ( $\mathrm{MH}$ and EB). At this stage, papers with no microbiological results were excluded. An extraction spreadsheet was used to 
collect data from all included papers. This included the number of patients, mean or median age, the percentage of positivity of pleural fluid culture and the absolute number of positive culture results for each organism/bacterial group.

The Strep. milleri group was combined with the viridans streptococci group to avoid overlap. Enterobacteriaceae were grouped under a single heading except for Klebsiella spp., as these were noted to be a site of difference between reports from different regions. All anaerobic bacteria were reported as a single group to avoid breaking down the categories into very small numbers.

\section{Data analysis}

There was no formal assessment for risk of bias in the studied papers, given that the main area of the review was based on observational data and not treatment effects. As a quality measure, papers with poorly reported microbiological results were excluded from the final dataset used to synthesise results. This was carried out independently by two reviewers ( $\mathrm{MH}$ and $\mathrm{RA}$ ) who analysed the full dataset to appraise the quality of the reporting of the microbiology results. Papers were judged to have good-quality data if culture results clearly reported the names of the organism or group and there was an account of results of anaerobic cultures (whether positive or negative). Any disagreement on the list of included papers was settled by discussion between reviewers.

Data regarding the age and number of study participants were reported as mean or median. Where appropriate, the mean of some variables was estimated from the median and range using the following formula: mean $=[(2 \times$ median $)+$ minimum + maximum $] / 4[11]$.

The absolute numbers of isolated organisms/groups from each study were summated. The sum of the numbers per organism/group was expressed as a percentage of the grand total of all organisms/groups across all studies.

\section{Subgroup analyses}

A pre hoc subgroup analysis of relative contribution of each organism was carried out according to 1) the latitude of the city where the publication came from; this was divided into three regions: temperate regions (north or south of latitude $40^{\circ}$ ), subtropical regions (between $23.5^{\circ}$ and $40^{\circ}$ north and south of the equator) and tropical regions (between $23.5^{\circ}$ north and $23.5^{\circ}$ south of the equator) [12]; 2) the setting of infection: relative contribution of different organisms in community-acquired versus hospital-acquired infections; and 3) time trend: reports were divided into two groups according to publication date. The first period was 2000-2008 and the second period was 2009-2017 to examine for changing trends in the microbiology by time.

\section{Results}

\section{Screening results}

The initial search identified 20980 publications. Titles/abstracts of these publications were screened for eligibility resulting in the exclusion of 20705 publications due to reasons detailed in the PRISMA diagram (figure 1). 211 full papers were downloaded and assessed for eligibility. Of these, 75 articles [2, 8-10, 13-83] were judged to have good-quality previously unpublished microbiological data and were included in the data synthesis. The reasons for exclusion of the remaining 136 papers are detailed in figure 1 . The repeat search between 2017 and 2018 yielded 536 publications, of which two were deemed potentially relevant, but on full analysis were excluded.

\section{Study characteristics}

The total number of patients reported from the 75 studies combined was 10241 . The mean of mean ages reported from the 56 studies that exclusively included adult patients was $54.4 \pm 9.5$ years. The characteristics of included studies are presented in table 1 .

\section{Synthesis of results}

Combining numbers of organisms described in all studies, a total of 6202 bacterial isolates were reported; 50.4\% (95\% CI 48-50.6\%) were Gram-positive aerobic organisms, 37.5\% (95\% CI 37.2-39.6\%) were Gram-negative aerobic organisms and $12.1 \%$ (95\% CI 11.4-13.1\%) were anaerobes. All studies used conventional culture techniques, except for a single study [36] where nucleic acid tests were used. The mean diagnostic yield of bacterial culture was 56\% (95\% CI 50.6-61.4\%).

The most common aerobic isolates were Staphylococcus aureus (20.7\%), viridans streptococci group (18.7\%), Pseudomonas spp. (17.6\%), Enterobacteriaceae group (11.9\%), Strep. pneumoniae (10.8\%), Klebsiella spp. (10.7\%), Acinetobacter species (5\%) and coagulase negative staphylococci (4.5\%) (figure 2). 


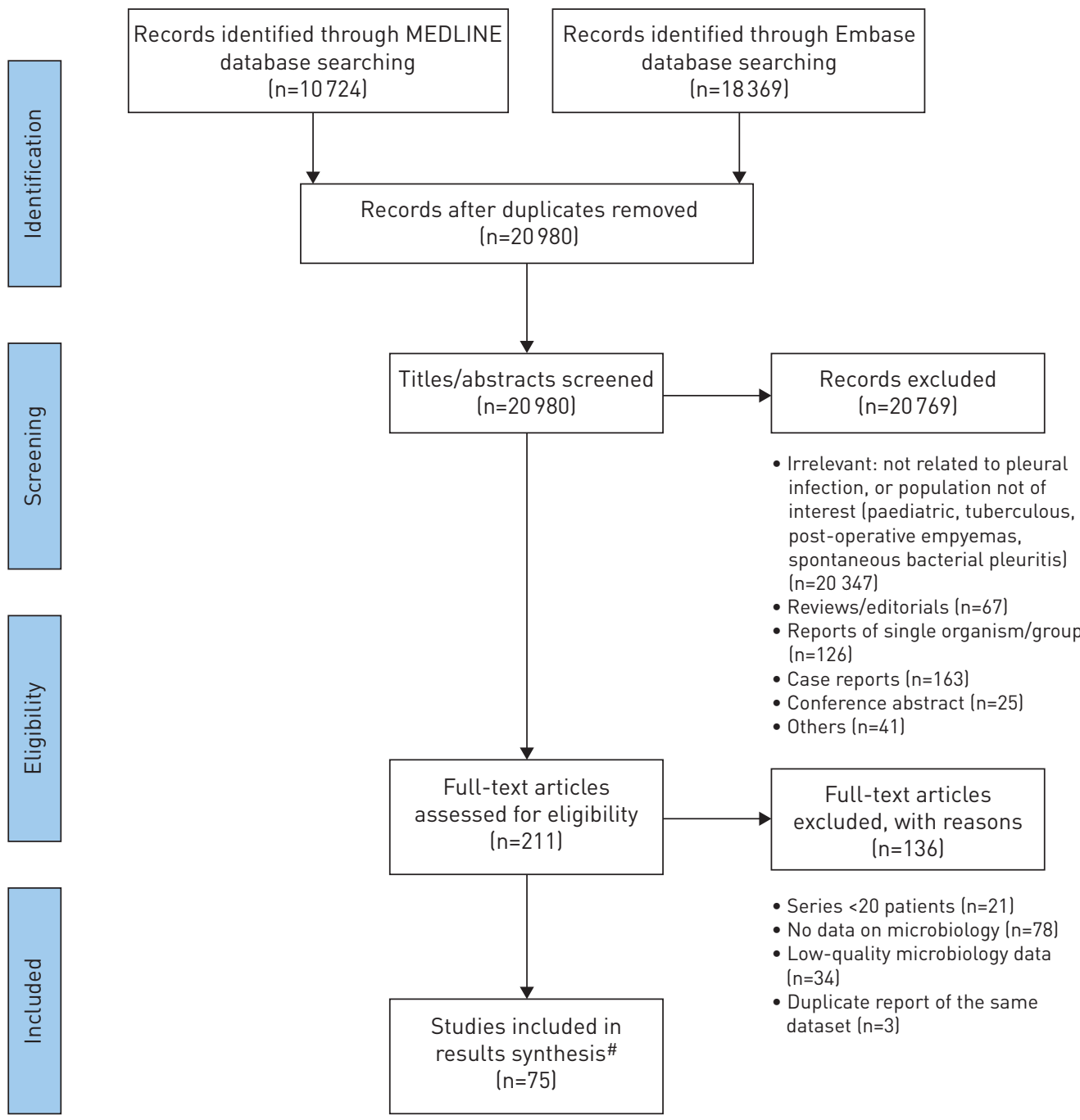

FIGURE 1 Preferred Reporting Items for Systematic Reviews and Meta-Analyses (PRISMA) flowchart of the systematic review. \#: French $\mathrm{n}=1$, Portuguese $n=1$, Lithuanian $n=1$, Korean $n=1$, Ukrainian $n=1$, English $n=70$.

\begin{tabular}{|c|c|}
\hline Age (mean of mean \pm sD of 56 studies) years ${ }^{\#}$ & $54.4 \pm 9.5$ \\
\hline \multicolumn{2}{|l|}{ Number of cases per publication } \\
\hline Median (range) & $83(20-601)$ \\
\hline Interquartile range & $47.5-160.75$ \\
\hline Total cases $\mathrm{n}$ & 10241 \\
\hline \multicolumn{2}{|l|}{ Study type } \\
\hline Observational & $45(59)$ \\
\hline Interventional & $30(41)$ \\
\hline \multicolumn{2}{|l|}{ Study nature } \\
\hline Medical & $58(78)$ \\
\hline Surgical & $16(22)$ \\
\hline \multicolumn{2}{|l|}{ Age by study cohort years } \\
\hline Medical & $58.8 \pm 9.2$ \\
\hline Surgical & $49.6 \pm 9.6$ \\
\hline Percentage positivity of microbial culture mean \pm so (weighted mean) & $56 \pm 21(52.4)$ \\
\hline
\end{tabular}

Data are presented as mean \pm SD or $\mathrm{n}(\%)$, unless otherwise stated. ${ }^{*}$ : studies with mixed adult and paediatric populations were not included. 

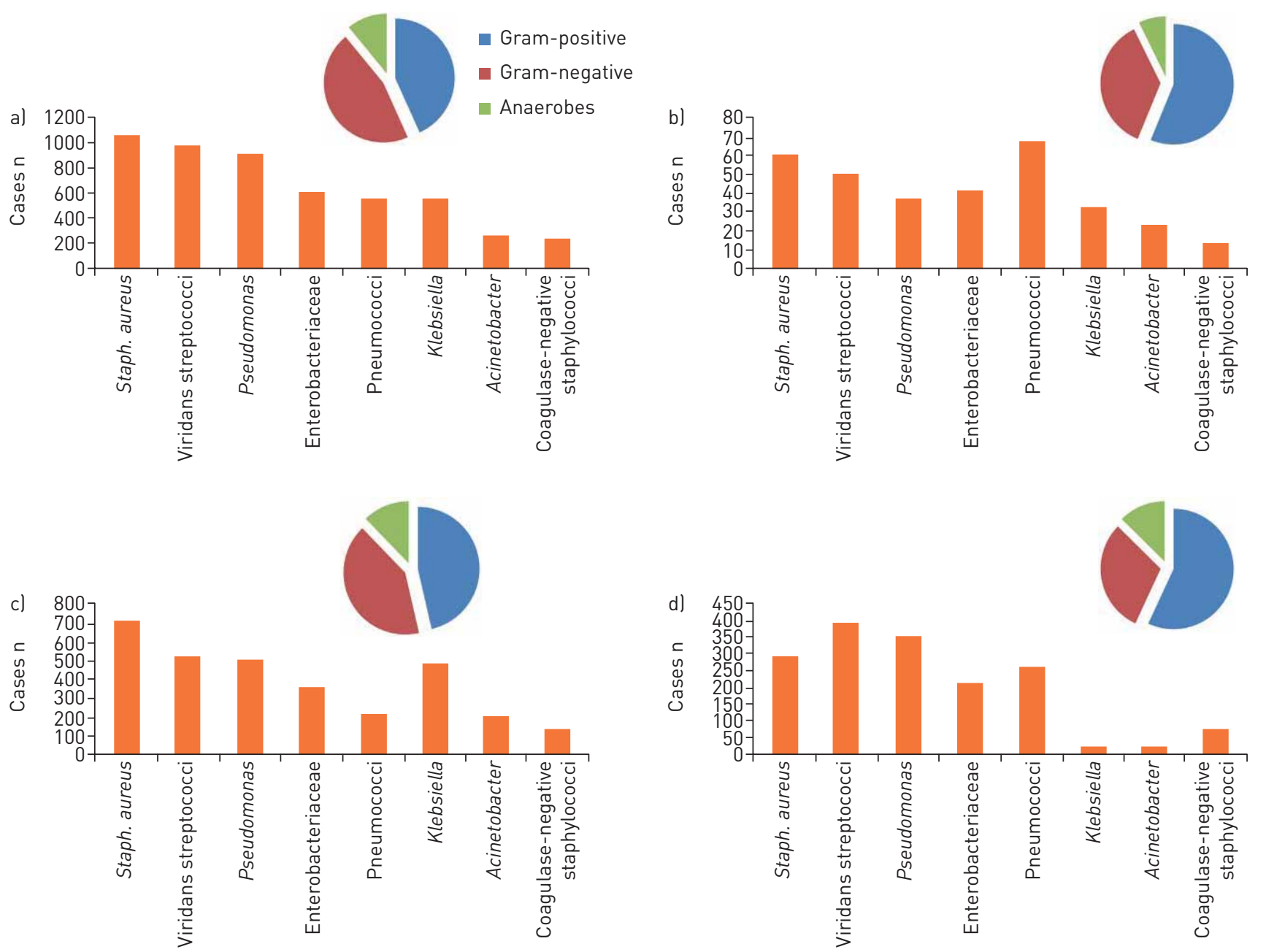

FIGURE 2 The relative contribution of organism categories (pie charts) and specific organisms (bar charts) in culture results of infected pleural fluid a) worldwide ( $\mathrm{n}=10241$ ); b) tropics (between $23.5^{\circ}$ north and $23.5^{\circ}$ south of the equator; $\mathrm{n}=903$ ); c) subtropics (between $23.5^{\circ}$ and $40^{\circ}$ north and south of the equator; $n=5556$ ); d) temperate regions (north or south of latitude $40^{\circ} ; n=3782$ ).

24 papers (reporting on 3842 patients) presented culture results separately for Strep. milleri and viridans streptococci group. From a total of 531 isolates, 309 (58.3\%) were from the milleri group.

Some of the studies reporting unselected results of pleural infection included results of cultures positive for mycobacterium tuberculosis (TB) or fungi. The pooled numbers from these reports suggest that the incidence of $\mathrm{TB}$ as an aetiology of suspected bacterial infection was $8.8 \%$ (in a total of 2074 cases from 15 studies), while that of fungi was 3\% (in a total of 3003 cases from 20 studies).

24 studies indicated the proportion of cultures that yielded more than one organism from the same sample. The median percentage of polymicrobial results from the overall cultured samples was $12.9 \%$ (interquartile range 8.0-17.9\%). Four of these studies provided details about the polymicrobial results. In $75 \%$ of the instances anaerobic bacteria were isolated, they were found to be mixed with other organisms, either aerobic or anaerobic. $49 \%$ of the isolates from the viridans group were retrieved from polymicrobial cultures in comparison to $28.5 \%$ for the Staph. aureus isolates. Notably, none of the pneumococci isolates grew in mixed cultures.

\section{Subgroup analyses}

Geographical differences

The majority of publications from the tropics came from the following countries: Thailand, Mexico, Singapore, Saudi Arabia and Cameroon. Most of the studies from the subtropics came from the northern hemisphere (96\%) and these were from South East Asia, southern US, the Middle East and southern Europe. All studies from temperate regions came from Europe and North America. 
Figure 2 shows the relative contribution of the different microbial groups and the preponderant aerobic organisms in each of the three geographical regions (the tropics, subtropics and temperate regions). The subtropics had higher incidence of Gram-negative organisms in comparison to the two other regions. Staph. aureus was the most preponderant culture result, followed by Klebsiella, Pseudomonas spp. and the viridans group, which were all more common than pneumococci. Both the tropics and temperate regions had a higher incidence of Gram-positive organisms. Viridans streptococci were the most widely reported organisms from temperate regions, while Strep. pneumoniae were the most commonly reported organisms from the tropics (figure 2)

25 articles (reporting on 4285 patients) presented data on the methicillin sensitivity of the Staph. aureus isolates. Supplementary figure S1 presents the proportions of the methicillin-sensitive Staph. aureus to the methicillin-resistant Staph. aureus isolated from the three geographical regions.

In terms of geographical differences in nonbacterial results, positive TB culture results were reported in 37 (9\%) out of 406 patients in the largest study reported from the United Kingdom [36], while positive TB cultures were reported in 148 (29\%) out of 511 patients in the largest two reports from India [38, 62].

\section{Community- versus hospital-acquired infection}

11 studies reporting data on 1523 patients identified the setting of the pleural infection. Figure 3 shows the combined numbers of culture isolates from these studies. In community-acquired infections, Gram-positive aerobes (65.1\%) were the predominant group, followed by anaerobes $(17.8 \%)$ and then Gram-negative aerobes (17.1\%). The most common aerobic isolates were the viridans group (32\%), pneumococci $(22 \%)$, followed by Staph. aureus (18.5\%). In hospital-acquired infections, Gram-negative aerobes had a larger share $(37.5 \%)$, with less anaerobic isolates (11\%). The most common aerobic isolates were Staph. aureus (37.8\%) followed by the Enterobacteriaceae group, Pseudomonas spp. and Klebsiella spp., which combined made up $26.7 \%$ of aerobic isolates. Information on resistance to methicillin in Staph. aureus isolates was included in these 11 studies. In community-acquired infections, $67 \%$ of the Staph. aureus isolates were methicillin-sensitive, while in the hospital-acquired group, $42 \%$ of the isolates were methicillin-sensitive.

\section{Time trends}

Supplementary figure S2 shows the contribution of the three main microbial groups in the aetiology of pleural infection in the earlier half (2000-2008) and the latter half (2009-2017) of the study period. The total number each of the 10 most common organisms was isolated in the two study periods is presented in supplementary figure S3. Supplementary figure S4 shows the proportion of methicillin-sensitive and -resistant Staph. aureus isolates according to the time period.
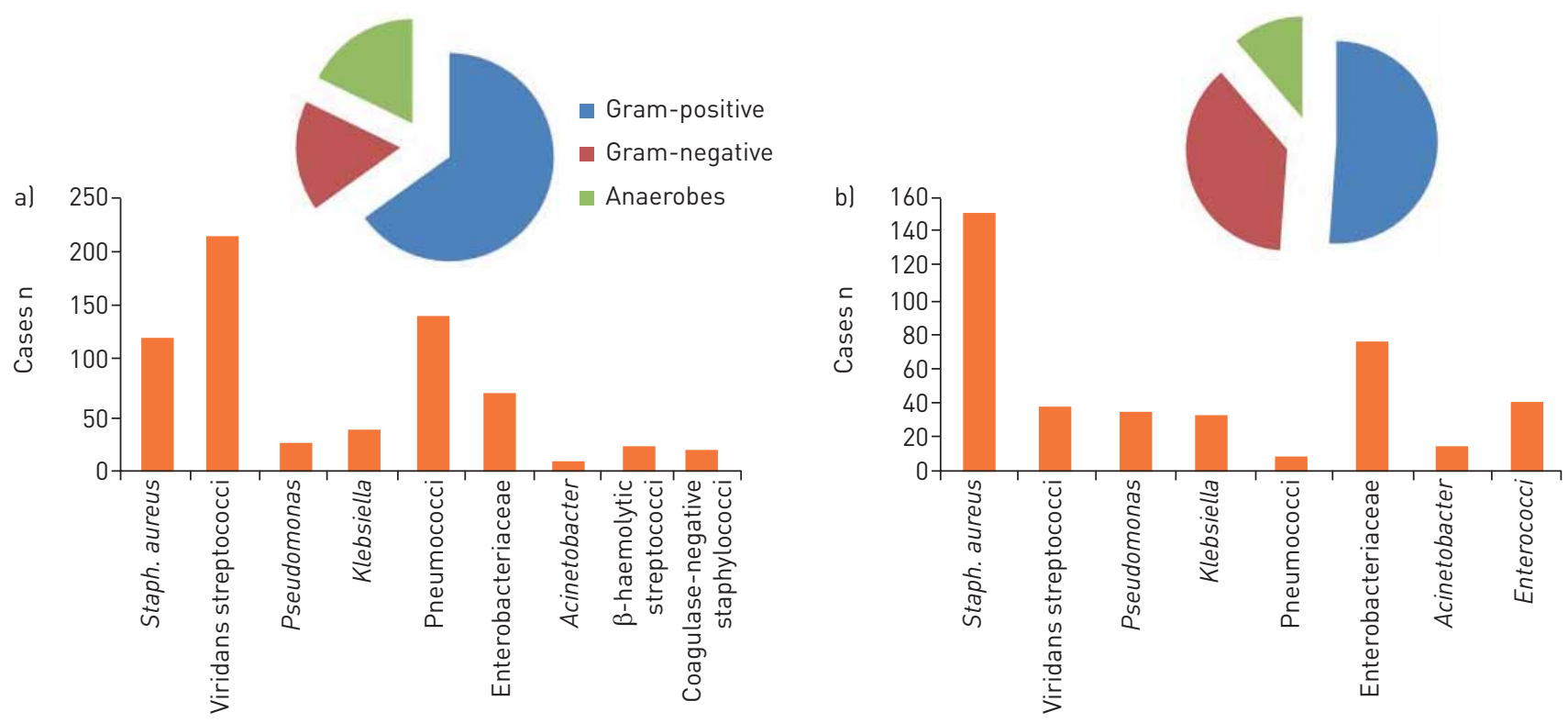

FIGURE 3 The relative contribution of organism categories (pie charts) and specific organisms (bar charts) in culture results of infected pleural fluid. $n=1523$ (11 studies). a) Community-acquired infection ( $n=1097)$; b) hospital-acquired infection ( $n=426$ ). 


\section{Discussion}

To our knowledge, this study is the first systematic review of microbiology of pleural infection in adults. The results demonstrate that the mean diagnostic yield of bacterial cultures on pleural fluid is 56\% (table 1), which means that in more than two-fifths of cases, the organism(s) remains unknown and antimicrobial treatment is entirely empirical. The information obtained from our data should inform clinical care, and specifically choice of empirical therapy by region.

In total, $12.9 \%$ of cultures demonstrated more than one isolate. Given that cultures identified the causative organisms in only about half of the instances, the incidence of polymicrobiality in culture-positive samples can be assumed to be double that figure: $\sim 23 \%$. In a metagenomic study by DYRHOVDEN et al. [84], massive parallel sequencing of bacterial DNA on 44 samples of pleural infection identified polymicrobial infection in $25 \%$ of the parapneumonic and $59 \%$ of the primary pleural infection samples.

The role of oropharyngeal flora, composed of strict or facultative anaerobes as pathogens causing pleural infection, is supported in this study. The viridans group and pneumococci are consistently among the most common isolates in different regions, and particularly in community-acquired infection. Isolates from the "salivarius" and mutans groups (which fall in the viridans group) were reported, and $\beta$-haemolytic streptococci was the sixth commonest isolate in community-acquired infections, stressing the significant role played by oropharyngeal flora in the likely pathogenesis of pleural infection. In the aforementioned metagenomic study, Strep. intermedius and Fusobacterium nucleatum (both commonly implicated in dental/periodontal infection) were the most common pathogens identified, particularly in non-parapenumonic pleural infections [84]. The exact mechanisms whereby oral flora gain access to the pleural space are incompletely understood, but this pattern seems independent of geography. The aforementioned study found that pneumococci are exclusively isolated from monomicrobial cultures, in contrast to the viridans streptococci that are commonly mixed with anaerobes [84]. This is similar to the findings of this review and further confirms that there is more than one pathobiological pathway for the development of pleural infection.

Strictly anaerobic bacteria are found in $12.8 \%$ of all culture-positive cases, and in $17.8 \%$ in community-acquired infections alone. Anaerobic organisms are known to be difficult to culture, with specific culture methods required [85], meaning that our data are probably an underestimate of the true contribution. In a study addressing anaerobic organisms in pleural infection where ideal culture methods were undertaken, anaerobic organisms were isolated in $74 \%$ of culture-positive pleural effusions [85], with micro-aerophilic streptococci (from the viridans group) included in these numbers. Anaerobic bacteria were found to be mixed with aerobic bacteria in almost two-thirds of cases, and three or more organisms were isolated from $14 \%$ of the samples [85]. These data highlight the importance of anaerobic treatment in empirical regimes.

The preponderance of oropharyngeal flora in culture results might be explained in cases of hospital-acquired pleural infection due to a presumed high risk of aspiration in this cohort of patients. However, the results of this review demonstrate that anaerobes were isolated relatively more commonly in community-acquired infections, which may be related to poor dental hygiene [86] with spread to the pleura via the haematogenous route. The tropism of anaerobes to the pleura is thought to be due to the favourable environment of the space [1] which is very different from the environment of the lung parenchyma which has high oxygen tension.

Staph. aureus is by far the most common organism isolated regardless of study or setting, but is particularly prevalent in hospital-acquired disease. Although many studies included are somewhat old, it is alarming that methicillin-resistant isolates represent one-third of positive cultures in community-acquired infection. This has important implications for the initial choice of empiric antibiotics.

A clear geographic variation in pleural infection was seen, although the precise reasons behind this strong signal are unclear. There is a large economic, particularly health economic, variation between the groups of countries making up the three regions, with different hospital-level numbers, availability and complexity of patient care, and different local antibiotic prescribing practices. Besides climatic variations, all these factors potentially contribute to variation to patterns of microbiology and antibiotic resistance.

In tropical regions, the profile was strongly Gram-positive, with pneumococci being the most common isolate. We speculate that this may be due to the generally younger populations affected by pleural infection in these regions, and relatively higher rates of HIV infection (particularly in Africa), which are both associated with higher incidence of pneumococcal pneumonia. The profile in the temperate regions was also strongly Gram-positive, with the highest proportion attributable to viridans streptoccoci. This group is particularly associated with oral/dental infections and poor oral hygiene [84], which could be related to the higher alcohol-related disease burden in North America and Western Europe (where most of the studies from temperate regions originate) in comparison to the South East Asia region [87]. 
In the subtropics, a higher proportion of Gram-negative isolates was seen. However, the results are confounded by a large number of reports from this region originating in Taiwan and South Korea, where life expectancy is among the highest in the world, translating to a higher comorbidity burden, and hence higher hospitalisations and hospital-acquired infections, which could account for the difference in the prevalence of Gram-negative pleural infections in this region. The peculiarity of the higher incidence of Klebsiella spp. from this region is an extension of an observed, and not completely explained, trend of increased propensity of the Klebsiella spp. to cause pyogenic infections in other parts of the body [88]. In addition, there is a high prevalence of Pseudomonas spp. infection, which is mainly driven by data from two studies from the Indian subcontinent $[43,62]$. There are data to suggest that higher temperatures are associated with increased incidence of Pseudomonas spp. and other Gram-negative bacterial bloodstream infections [89].

The analysis by the publication year shows an increased role played by Gram-positive bacteria in the past few years. In particular, Staph. aureus overtakes the viridans streptococci as the most common isolate, and the proportion of methicillin-resistant isolates increases from $48 \%$ to $58 \%$. This pattern is not uniform across regions, as methicillin resistance appears highest in the subtropics.

There are limitations to this study; there existed a large heterogeneity in reporting bacterial groups, particularly organisms from the Streptococcus genus. Despite attempts to exclude paediatric patients and those with post-operative pleural infections, several reports included some patients from these categories, which decreases the accuracy of the data to a degree. Information on the site of acquiring infection (community versus hospital) was not available in many of the included studies, which could have contributed to a degree to the temporal and regional variations noticed. The majority of the included papers relied on conventional cultures in delineating the microbial aetiology, which means that the burden of infection caused by difficult-to-culture bacteria is likely to be underestimated. Finally, there was a predominance of reports from Europe and the Far East and a relative paucity of reports from Africa and the South America, which affects the reliability of the findings to a degree.

\section{Conclusion}

In pleural infection, the geographical location and the setting of infection seem to have a bearing on the expected causative organisms. This should be reflected in the choice of empirical antimicrobial treatment to address the preponderance of certain microbes and prevalence of antibiotic resistance, which should always be supported by data on local resistance patterns.

Support statement: M. Hassan is a recipient of the European Respiratory Society long-term research fellowship (ERS 2016 7333). T. Cargill is funded as an Academic Clinical Fellow by the National Institute of Health Research and by a Wellcome Trust PhD Training Fellowship for Clinicians (grant number 211042/Z/18/Z). Funding information for this article has been deposited with the Crossref Funder Registry.

Contribution details: J.P. Corcoran, I. Psallidas and N.M. Rahman conceived the review. E. Harriss and M. Hassan performed the literature search. M. Hassan, J.P. Corcoran, R. Asciak, R.M. Mercer and N.M. Rahman discussed and agreed on inclusion and exclusion criteria. M. Hassan, T. Cargill, R. Asciak and E.O. Bedawi screened publications for eligibility. M. Hassan and T. Cargill extracted, combined and analysed data. M. Hassan wrote the first version of the manuscript. N.M. Rahman, J.P. Corcoran and R.M. Mercer critically reviewed the manuscript. All authors reviewed and approved the final manuscript.

Conflict of interest: None declared.

\section{References}

1 Bedawi EO, Hassan M, Rahman NM. Recent developments in the management of pleural infection: a comprehensive review. Clin Respir J 2018; 12: 2309-2320.

2 Rahman NM, Maskell NA, West A, et al. Intrapleural use of tissue plasminogen activator and DNase in pleural infection. N Engl J Med 2011; 365: 518-526.

3 Piccolo F, Pitman N, Bhatnagar R, et al. Intrapleural tissue plasminogen activator and deoxyribonuclease for pleural infection. An effective and safe alternative to surgery. Ann Am Thorac Soc 2014; 11: 1419-1425.

4 Shen H-N, Lu C-L, Li C-Y. Epidemiology of pleural infections in Taiwan from 1997 through 2008: pleural infections in Taiwan. Respirology 2012; 17: 1086-1093.

5 Davies HE, Davies RJO, Davies CWH, et al. Management of pleural infection in adults: British Thoracic Society pleural disease guideline 2010. Thorax 2010; 65: ii41-ii53.

6 Lisboa T, Waterer GW, Lee YCG. Pleural infection: changing bacteriology and its implications. Respirology 2011; 16: 598-603.

7 Wrightson JM, Wray JA, Street TL, et al. Absence of atypical pathogens in pleural infection. Chest 2015; 148: e102-e103.

8 Falguera M, Carratalà J, Bielsa S, et al. Predictive factors, microbiology and outcome of patients with parapneumonic effusion. Eur Respir J 2011; 38: 1173-1179.

9 Tu C-Y, Hsu W-H, Hsia T-C, et al. The changing pathogens of complicated parapneumonic effusions or empyemas in a medical intensive care unit. Intensive Care Med 2006; 32: 570-576. 
10 Lin $\mathrm{Y}-\mathrm{C}, \mathrm{Tu} \mathrm{C}-\mathrm{Y}$, Chen W, et al. An urgent problem of aerobic Gram-negative pathogen infection in complicated parapneumonic effusions or empyemas. Intern Med 2007; 46: 1173-1178.

11 Hozo SP, Djulbegovic B, Hozo I. Estimating the mean and variance from the median, range, and the size of a sample. BMC Med Res Methodol 2005; 5: 13.

12 American Meteorological Society. Meteorology Glossary. http://glossary.ametsoc.org/wiki/Main_Page Date last updated: June 26, 2018.

13 LeMense GP, Strange C, Sahn SA. Empyema thoracis. Therapeutic management and outcome. Chest 1995; 107: 1532-1537.

14 Wait MA, Sharma S, Hohn J, et al. A randomized trial of empyema therapy. Chest 1997; 111: 1548-1551.

15 Lim TK, Chin NK. Empirical treatment with fibrinolysis and early surgery reduces the duration of hospitalization in pleural sepsis. Eur Respir J 1999; 13: 514-518.

16 Davies CW, Kearney SE, Gleeson FV, et al. Predictors of outcome and long-term survival in patients with pleural infection. Am J Respir Crit Care Med 1999; 160: 1682-1687.

17 Odeh M, Sabo E, Oliven A, et al. Role of tumor necrosis factor- $\alpha$ in the differential diagnosis of parapneumonic effusion. Int J Infect Dis 2000; 4: 38-41.

18 Chen KY, Hsueh PR, Liaw YS, et al. A 10-year experience with bacteriology of acute thoracic empyema: emphasis on Klebsiella pneumoniae in patients with diabetes mellitus. Chest 2000; 117: 1685-1689.

19 Kalaajieh WK. Etiology of exudative pleural effusions in adults in North Lebanon. Can Respir J 2001; 8: 93-97.

20 Chu MW, Dewar LR, Burgess JJ, et al. Empyema thoracis: lack of awareness results in a prolonged clinical course. Can J Surg 2001; 44: 284-288.

21 Tuncozgur B, Ustunsoy H, Sivrikoz MC, et al. Intrapleural urokinase in the management of parapneumonic empyema: a randomised controlled trial. Int J Clin Pract 2001; 55: 658-660.

22 Hoth JJ, Burch PT, Bullock TK, et al. Pathogenesis of posttraumatic empyema: the impact of pneumonia on pleural space infections. Surg Infect 2003; 4: 29-35.

23 Anstadt MP, Guill CK, Ferguson ER, et al. Surgical versus nonsurgical treatment of empyema thoracis: an outcomes analysis. Am J Med Sci 2003; 326: 9-14.

24 Petrakis IE, Kogerakis NE, Drositis IE, et al. Video-assisted thoracoscopic surgery for thoracic empyema: primarily, or after fibrinolytic therapy failure? Am J Surg 2004; 187: 471-474.

25 Diacon AH, Theron J, Schuurmans MM, et al. Intrapleural streptokinase for empyema and complicated parapneumonic effusions. Am J Respir Crit Care Med 2004; 170: 49-53.

26 Banga A, Khilnani G, Sharma S, et al. A study of empyema thoracis and role of intrapleural streptokinase in its management. BMC Infect Dis 2004; 4: 19.

27 Luh S-P, Chou M-C, Wang L-S, et al. Video-assisted thoracoscopic surgery in the treatment of complicated parapneumonic effusions or empyemas: outcome of 234 patients. Chest 2005; 127: 1427-1432.

28 Khwaja S, Rosenbaum DH, Paul MC, et al. Surgical treatment of thoracic empyema in HIV-infected patients: severity and treatment modality is associated with CD4 count status. Chest 2005; 128: 246-249.

29 Tsai T-H, Jerng J-S, Chen K-Y, et al. Community-acquired thoracic empyema in older people. J Am Geriatr Soc 2005; 53: 1203-1209.

30 Lin F, Chen $\mathrm{Y}$, Chen $\mathrm{F}$, et al. Cytokines and fibrinolytic enzymes in tuberculous and parapneumonic effusions. Clin Immunol 2005; 116: 166-173.

31 Cheng G, Vintch JRE. A retrospective analysis of the management of parapneumonic empyemas in a county teaching facility from 1992 to 2004. Chest 2005; 128: 3284-3290.

32 Ozol D, Oktem S, Erdinc E. Complicated parapneumonic effusion and empyema thoracis: microbiologic and therapeutic aspects. Respir Med 2006; 100: 286-291.

33 Bilgin M, Akcali Y, Oguzkaya F. Benefits of early aggressive management of empyema thoracis. ANZ J Surg 2006; 76: $120-122$

34 Smolikov A, Smolyakov R, Riesenberg K, et al. Prevalence and clinical significance of pleural microbubbles in computed tomography of thoracic empyema. Clin Radiol 2006; 61: 513-519.

35 Ahmed RA, Marrie TJ, Huang JQ. Thoracic empyema in patients with community-acquired pneumonia. Am J Med 2006; 119: 877-883.

36 Maskell NA, Batt S, Hedley EL, et al. The bacteriology of pleural infection by genetic and standard methods and its mortality significance. Am J Respir Crit Care Med 2006; 174: 817-823.

37 Jiménez D, Díaz G, García-Rull S, et al. Routine use of pleural fluid cultures. Are they indicated? Limited yield, minimal impact on clinical decisions. Respir Med 2006; 100: 2048-2052.

38 Malhotra P, Aggarwal AN, Agarwal R, et al. Clinical characteristics and outcomes of empyema thoracis in 117 patients: a comparative analysis of tuberculous vs. non-tuberculous aetiologies. Respir Med 2007; 101: 423-430.

39 Levinson GM, Pennington DW. Intrapleural fibrinolytics combined with image-guided chest tube drainage for pleural infection. Mayo Clin Proc 2007; 82: 407-413.

40 Tsang KY, Leung WS, Chan VL, et al. Complicated parapneumonic effusion and empyema thoracis: microbiology and predictors of adverse outcomes. Hong Kong Med J 2007; 13: 178-186.

41 Kim Y-J, Cha S-I, Kwon J-S, et al. Treatment results and prognostic factors of complicated parapneumonic effusion and empyema. Tuberc Respir Dis 2007; 63: 24-30.

42 Chen $\mathrm{C}-\mathrm{H}, \mathrm{Hsu} \mathrm{W}-\mathrm{H}$, Chen $\mathrm{H}-\mathrm{J}$, et al. Different bacteriology and prognosis of thoracic empyemas between patients with chronic and end-stage renal disease. Chest 2007; 132: 532-539.

43 Mohanty S, Kapil A, Das BK. Bacteriology of parapneumonic pleural effusions in an Indian hospital. Trop Doct 2007; 37: 228-229.

44 Potaris K, Mihos P, Gakidis I, et al. Video-thoracoscopic and open surgical management of thoracic empyema. Surg Infect 2007; 8: 511-518.

45 El Solh AA, Alhajjhasan A, Ramadan FH, et al. A comparative study of community- and nursing home-acquired empyema thoracis. J Am Geriatr Soc 2007; 55: 1847-1852.

46 Liang SJ, Chen W, Lin Y-C, et al. Community-acquired thoracic empyema in young adults. South Med J 2007; 100: $1075-1080$.

47 Wozniak CJ, Paull DE, Moezzi JE, et al. Choice of first intervention is related to outcomes in the management of empyema. Ann Thorac Surg 2009; 87: 1525-1530. 
48 Lin $\mathrm{M}-\mathrm{C}$, Chen $\mathrm{Y}-\mathrm{C}$, Wu J-T, et al. Diagnostic and prognostic values of pleural fluid procalcitonin in parapneumonic pleural effusions. Chest 2009; 136: 205-211.

49 Chalmers JD, Singanayagam A, Murray MP, et al. Risk factors for complicated parapneumonic effusion and empyema on presentation to hospital with community-acquired pneumonia. Thorax 2009; 64: 592-597.

50 Chen $\mathrm{C}-\mathrm{H}$, Chen $\mathrm{W}$, Chen $\mathrm{H}-\mathrm{J}$, et al. Transthoracic ultrasonography in predicting the outcome of small-bore catheter drainage in empyemas or complicated parapneumonic effusions. Ultrasound Med Biol 2009; 35: 1468-1474.

51 Chen W, Lin Y-C, Liang S-J, et al. Hospital-acquired thoracic empyema in adults: a 5-year study. South Med J 2009; 102: 909-914.

52 Kundu S Mitra S, Mukherjee S, et al. Adult thoracic empyema: a comparative analysis of tuberculous and nontuberculous etiology in 75 patients. Lung India 2010; 27: 196-201.

53 Zablockis R, Petruskeviciene R, Nargela RV. Pleuros empiemos ir komplikuoto parapneumoninio pleurito priežastys ir rizikos veiksniai. [Causes and risk factors of pleural empyema and complicated parapneumonic pleural effusion]. Medicina 2010; 46: 113-119.

54 Metin M, Yeginsu A, Sayar A, et al. Treatment of multiloculated empyema thoracis using minimally invasive methods. Singapore Med J 2010; 51: 242-246.

55 Bar I, Stav D, Fink G, et al. Thoracic empyema in high-risk patients: conservative management or surgery? Asian Cardiovasc Thorac Ann 2010; 18: 337-343.

56 Herrera-Kiengelher L, Báez-Saldaña R, Salas-Hernández J, et al. Frequency of adverse events and mortality in patients with pleural empyema in a public referral hospital in Mexico City. Int J Tuberc Lung Dis 2010; 14: 1187-1192.

57 Meyer CN, Rosenlund S, Nielsen J, et al. Bacteriological aetiology and antimicrobial treatment of pleural empyema. Scand J Infect Dis 2011; 43: 165-169.

58 Monteiro R, Alfaro T, Correia L, et al. Abcesso pulmonar e empiema torácico: análise retrospectiva num serviço de medicina interna. [Lung abscess and thoracic empyema: retrospective analysis in an internal medicine department]. Acta Med Port 2011; 24: Suppl 2: 229-240.

59 Marks DJB, Fisk MD, Koo CY, et al. Thoracic empyema: a 12-year study from a UK tertiary cardiothoracic referral centre. PLoS One 2012; 7: e30074.

60 Pefura Yone EW, Kuaban C, Afane Ze E, et al. Caractéristiques cliniques, bactériologiques et évolutives de la pleurésie purulente non-tuberculeuse chez les patients adultes séropositifs et séronégatifs au VIH à Yaoundé, Cameroun [Clinical characteristics, microbiologic findings and outcome of non-tuberculous purulent pleural effusion in HIV-infected and non HIV-infected adult patients in Yaounde, Cameroon]. Rev Pneumol Clin 2012; 68: $225-232$

61 Považan A, Vukelić A, Kurucin T, et al. The most common isolates from pleural infections. Acta Microbiol Immunol Hung 2012; 59: 375-385.

62 Sonali J. Empyema thoracis: bacteriological analysis of pleural fluid from the largest chest hospital in Delhi. IOSR J Dent Med Sci 2013; 3: 46-51.

63 White HD, White BAA, Song J, et al. Pleural infections: a 9-year review of bacteriology, case characteristics and mortality. Am J Med Sci 2013; 345: 349-354.

64 O'Connor JV, Chi A, Joshi M, et al. Post-traumatic empyema: aetiology, surgery and outcome in 125 consecutive patients. Injury 2013; 44: 1153-1158.

65 Kim S-K, Kang CU, Song SH, et al. Factors predictive of the failure of medical treatment in patients with pleural infection. Korean J Intern Med 2014; 29: 603-612

66 Chen K-C, Chen H-Y, Lin J-W, et al. Acute thoracic empyema: clinical characteristics and outcome analysis of video-assisted thoracoscopic surgery. I Formos Med Assoc 2014; 113: 210-218.

67 Ohuchi M, Inoue S, Ozaki Y, et al. Single-trocar thoracoscopy under local anesthesia for pleural space infection. Gen Thorac Cardiovasc Surg 2014; 62: 503-510.

68 Okiror L, Coltart C, Bille A, et al. Thoracotomy and decortication: impact of culture-positive empyema on the outcome of surgery. Eur J Cardiothorac Surg 2014; 46: 901-906.

69 Hajjar W, Ahmed I, Al-Nassar S, et al. Video-assisted thoracoscopic decortication for the management of late stage pleural empyema, is it feasible? Ann Thorac Med 2016; 11: 71-78.

70 Mehta HJ, Biswas A, Penley AM, et al. Management of intrapleural sepsis with once daily use of tissue plasminogen activator and deoxyribonuclease. Respiration 2016; 91: 101-106.

71 Kim J, Park JS, Cho Y-J, et al. Predictors of prolonged stay in patients with community-acquired pneumonia and complicated parapneumonic effusion. Respirology 2016; 21: 164-171.

72 Tsai C-H, Lai Y-C, Chang S-C, et al. Video-assisted thoracoscopic surgical decortication in the elderly with thoracic empyema: five years' experience. J Chin Med Assoc 2016; 79: 25-28.

73 Tronina EY, Shipulin PP, Baydan VI, et al. [Possibilities of modern technologies in the treatment of pleural empyema]. Klin Khir 2016: 46-48.

74 Chubar IV. [Analysis of microflora of pleural cavity in pleural empyema]. Klin Khir 2016; 83: 47-49.

75 Wong D, Yap E. Pleural infection in a New Zealand centre: high incidence in Pacific people and RAPID score as a prognostic tool. Intern Med J 2016; 46: 703-709.

76 Park C-K, Oh H-J, Choi H-Y, et al. Microbiological characteristics and predictive factors for mortality in pleural infection: a single-center cohort study in Korea. PLoS One 2016; 11: e0161280.

77 Majid A, Kheir F, Folch A, et al. Concurrent intrapleural instillation of tissue plasminogen activator and DNase for pleural infection. A single-center experience. Ann Am Thorac Soc 2016; 13: 1512-1518.

78 Porcel JM, Esquerda A, Vives M, et al. Etiology of pleural effusions: analysis of more than 3,000 consecutive thoracenteses. Arch Bronconeumol 2014; 50: 161-165.

79 Tantraworasin A, Thepbunchonchai A, Siwachat S, et al. Factors associated with recurrent bacterial empyema thoracis. Asian J Surg 2018; 41: 313-320.

80 Hsiao C-H, Chen K-C, Chen J-S. Modified single-port non-intubated video-assisted thoracoscopic decortication in high-risk parapneumonic empyema patients. Surg Endosc 2017; 31: 1719-1727.

81 Popowicz N, Bintcliffe O, De Fonseka D, et al. Dose de-escalation of intrapleural tissue plasminogen activator therapy for pleural infection. The Alteplase Dose Assessment for Pleural Infection Therapy Project. Ann Am Thorac Soc 2017; 14: 929-936. 
82 Asai N, Suematsu H, Hagihara M, et al. The etiology and bacteriology of healthcare-associated empyema are quite different from those of community-acquired empyema. J Infect Chemother 2017; 23: 661-667.

83 Koma Y, Inoue S, Oda N, et al. Clinical characteristics and outcomes of patients with community-acquired, health-care-associated and hospital-acquired empyema. Clin Respir J 2017; 11: 781-788.

84 Dyrhovden R, Nygaard RM, Patel R, et al. The bacterial aetiology of pleural empyema. A descriptive and comparative metagenomic study. Clin Microbiol Infect 2019; 25: 981-986.

85 Boyanova L, Djambazov V, Gergova G, et al. Anaerobic microbiology in 198 cases of pleural empyema: a Bulgarian study. Anaerobe 2004; 10: 261-267.

86 Corcoran JP, Wrightson JM, Belcher E, et al. Pleural infection: past, present, and future directions. Lancet Respir Med 2015; 3: 563-577.

87 James SL, Abate D, Abate $\mathrm{KH}$, et al. Global, regional, and national incidence, prevalence, and years lived with disability for 354 diseases and injuries for 195 countries and territories, 1990-2017: a systematic analysis for the Global Burden of Disease Study 2017. Lancet 2018; 392: 1789-1858.

88 Lin Y-T, Chen T-L, Siu LK, et al. Clinical and microbiological characteristics of community-acquired thoracic empyema or complicated parapneumonic effusion caused by Klebsiella pneumoniae in Taiwan. Eur J Clin Microbiol Infect Dis 2010; 29: 1003-1010.

89 Richet H. Seasonality in Gram-negative and healthcare-associated infections. Clin Microbiol Infect 2012; 18: 934-940. 